\title{
VALIDACIÓN DE UN INSTRUMENTO QUE ESTIMA LA INCIDENCIA DEL APRENDIZAJE COOPERATIVO EN EL ESTATUS SOCIOMÉTRICO DE LOS ALUMNOS
}

\author{
Esther Ruiz Palomo \\ Isabel Luis Rico \\ Tamara De La Torre Cruz \\ M. Camino Escolar Llamazares \\ Jonathan Huelmo García \\ Carmen Palmero Cámara \\ Alfredo Jiménez Eguizábal \\ Universidad de Burgos
}

RESUMEN: La dinámica social establecida en la actualidad demanda de los docentes la constante actualización a través de formación. Uno de los ámbitos formativos se centra en el desarrollo e implementación dentro del aula de metodologías activas que permitan al alumno construir su propio aprendizaje. El Aprendizaje Cooperativo (AC) incide de forma directa en la construcción propia del aprendizaje desde la dimensión social, ya que es en este ámbito donde tienen cabida los procesos de aprendizaje. Nuestra investigación se centra en la influencia del AC en el estatus sociométrico del alumnado de educación primaria y secundaria. Para ello se ha desarrollado una herramienta que permite delimitar el impacto de esta metodología activa a través de la variación producida en el estatus sociométrico del alumno dentro del grupo clase. Se presenta aquí el proceso de validación de la herramienta desarrollada.

PALABRAS CLAVE: aprendizaje cooperativo, metodologías activas, desempeño docente, sociograma (test sociométrico).

\section{COOPERATIVE LEARNING AND SOCIOMETRIC STATUS OF STUDENTS. A QUESTIONNAIRE VALIDATION PROCESS}

\footnotetext{
ABSTRACT: The social dynamics established at present demand of the teachers the constant updating through training. One of the training areas focuses on the development and implementation within the classroom of active methodologies that allow students to build their own learning. In the case of
} 
Cooperative Learning $(\mathrm{CL})$, it directly affects the construction of learning from the social dimension, since it is in this field that learning processes have a place. Our research focuses on the influence of $\mathrm{CL}$ on the sociometric status of students of primary education and secondary. To do this we have developed a tool that allows us to delimit the impact of this active methodology through the variation produced in the student's sociometric status within the class group. It is presented here the validation process of the tool developed.

KEYWORDS: Cooperative Learning, Active Methodologies, Teaching Performance, Sociogram (sociometric test).

Recibido: 21/12/2018

Aceptado: 12/04/2019

Correspondencia: Esther Ruiz Palomo, Universidad de Burgos, Facultad de Educación, Calle de Villadiego, 1, 09001 Burgos. Email: erpalomo@ubu.es.

\section{INTRODUCCIÓN}

Los nuevos retos a los que se enfrenta el contexto educativo hacen necesario replantear los procesos, de tal forma que se produzca un avance hacia paradigmas humanistas en los que la adquisición de competencias del Siglo XXI (Reimers y Chung, 2017), se dirija a formar ciudadanos capaces de promover el cambio social. En este sentido, las reformas del sistema educativo se han centrado en la incorporación de nuevos procedimientos que den respuesta a los objetivos centrados en promover una educación inclusiva, equitativa y de calidad (UNESCO, 2018). Investigaciones recientes muestran la relación positiva que se establece entre la introducción de estrategias que favorecen la interacción, el aprendizaje efectivo y la mejora en el clima de convivencia, siendo ejemplo de este tipo de estrategia el Aprendizaje Cooperativo (AC) (Johnson y Johnson, 2014), así como también la importancia de estos cambios estratégicos en la adquisición de las competencias.

El AC es un término genérico que se refiere a las técnicas utilizadas en la organización del aula dentro de los procesos de aprendizaje. Como tal, nos podemos retrotraer en la historia de la educación encontrándola como técnica utilizada durante diferentes épocas, no obstante, en los últimos veinte años se ha incrementado su uso debido a la mejora que produce en los procesos educativos (Gillies, 2014). Para denominarse AC los equipos cooperativos han de cumplir cuatro principios básicos (PIES): Positive interdependence- interdependencia positiva -; Individual accountability -responsabilidad individual-; Equal participation -participación igualitaria-; Simultaneous interaction -interacción simultánea- (Goikoetxea y Pascual, 2002; Kagan, 1995; Pliego, 2011).

Este auge en la implementación del AC dentro de las aulas va ligado al avance de las perspectivas teóricas sobre los efectos del uso de esta metodología en los estudiantes. Slavin $(2010,2013,2014)$ identifica cuatro de las principales teorías sobre el impacto del AC en los alumnos: motivacional, cohesión social, desarrollo cognitivo y elaboración cognitiva. Las teorías con mayor número de investigaciones empíricas son las ligadas al aspecto moti- 
vacional de los alumnos, no obstante, como aspecto relacionado, surge la cohesión social del grupo, fundamentada a su vez en la teoría del contacto (Goikoetxea y Pascual, 2002).

La teoría centrada en la cohesión social determina que en el AC se generan ocasiones en las que los alumnos cooperan dentro del grupo y se ayudan de forma intragrupal porque establecen vínculos sociales de ayuda y empatía, no por la consecución del objetivo final; también vincula el rendimiento académico del alumnado con la calidad de interacción del grupo (Slavin, 2014).

De esta forma, el AC se presenta como una metodología esencial para mejorar las relaciones dentro del ámbito educativo (Antolín, Martín-Pérez y Barbá, 2012; León del Barco, Polo del Río y Gonzalo Delgado, 2016; Polo del Río, Mendo Lázaro, Fajardo Bullón y León del Barco, 2017). Por lo tanto, se sitúa dentro de la acción para la mejora del clima de aula y de las relaciones interpersonales entre iguales, o peer relations, lo que también se vincula con el desarrollo cognitivo y el ajuste escolar (Martínez Lírola, 2016; 2016; Vega Vaca, Vidal Rodríguez y del Pilar García, 2013).

En este contexto el profesorado se presenta como elemento fundamental en el proceso de aplicación de estrategias de AC ya que en él reside la responsabilidad de cumplir con los cuatro principios básicos (PIES) y de velar por el correcto desarrollo de la implementación de esta metodología. Ante esta situación se hace necesario el desarrollo en el profesorado de competencias a través de la formación continua.

Nuestro trabajo se enmarca dentro del proyecto de investigación denominado "Metodologías Activas y Desempeño Docente: un camino para la inclusión en las aulas" del Grupo de Innovación Docente APAC de la Universidad de Burgos. El objetivo principal de dicho proyecto es evaluar el impacto de la utilización del AC en la mejora del clima de aula y del desempeño docente. Para ello se seleccionaron centros escolares cuyo profesorado participa en acciones de formación continua organizadas por el Centro de Formación e Innovación Educativa (CFIE), dirigidas a implantar esta metodología en sus aulas, así como en la interacción intragrupal del aula antes de la implantación del AC y tras su desarrollo. Este proyecto fue aprobado por la Dirección Provincial de Educación de Burgos, quien autorizó el acceso a los centros.

En la determinación de la interacción grupal se ha seleccionado el análisis sociométrico, ya que es una potente herramienta conceptual y metodológica que permite estudiar la vida de los grupos a partir de las fuerzas de atracción y rechazo entre sus miembros (Muñoz, Moreno y Jiménez, 2008). La teoría sobre el rechazo y la aceptación dentro del ámbito educativo está relacionada con el clima que se establece dentro del contexto del aula. La aceptación social entre pares está asociada con la adaptación, y ésta a su vez con el desarrollo socioemocional y académico, mientras que el rechazo es un factor de riesgo para la desadaptación presente y futura (García Bacete, Sureda García y Monjas Casares, 2010; Sureda García, García-Bacete y Monjas Casares, 2009; Rohner y Carrasco, 2014).

A través de la distinción de las fuerzas de aceptación y rechazo podemos establecer el estatus sociométrico de cada uno de los miembros, evaluando la integración social de los alumnos en sus aulas. Según Coie, Dodge y Coppotelli (1982), se distinguen cinco estatus sociométricos: popular, rechazado, aislado, controvertido y 
normal, los cuales se establecen a partir de la estandarización de los índices de elecciones y rechazos dentro de cada clase.

Para inferir el estatus sociométrico se puede utilizar la técnica de nominación intragrupal, de manera independiente, pidiendo a cada miembro del grupo un número limitado o ilimitado de nominaciones positivas o aceptaciones y de nominaciones negativas o rechazos, lo que nos acerca a la estructura interna. La suma de nominaciones positivas recibidas por cada miembro del grupo es una medida de la aceptación del grupo y la suma del número de nominaciones negativas es una medida de rechazo (García- Bacete, 2007, 2008; Muñoz, Moreno y Jiménez, 2008).

En nuestro caso, puesto que no existe un cuestionario que contemple las tres vertientes: afectiva, académica y social que interesan para la investigación, se hacía necesario desarroIlar una herramienta para determinar la interacción intragrupal previa a la implementación de la metodología de AC (pre- intervención) y tras el proceso de formación del docente e implantación del mismo en el aula (post- intervención), para captar las variaciones en la estructura interna a través de los estatus sociométricos. Lo que presentamos a continuación es el proceso de validación y aplicación de la herramienta desarrollada ad hoc.

\section{Método}

El paradigma interpretativo es el que centra nuestro trabajo a través de una metodología mixta que aúna herramientas y análisis de tipo cuantitativo y cualitativo para dar satisfacción al objetivo planteado.

Como en cualquier proceso de elaboración y validación de instrumentos de recolección de información, el primer paso fue identificar el objetivo general y las dimensiones que incluye, siendo la operacionalización la traducción en elementos medibles (Ruiz, 2014). Los constructos sociométricos, al medir procesos dinámicos, son sensibles al contexto y al grupo en el que se aplican, por lo que se hace necesario reflexionar sobre las preguntas planteadas.

\section{Objetivo}

Validar un cuestionario creado ad hoc para estimar la incidencia del aprendizaje cooperativo en el estatus sociométrico de determinadas aulas de educación primaria y secundaria.

\section{INSTRUMENTO}

Debido al contexto escolar en el que se desarrolla y el carácter grupal de la metodología de AC, los ámbitos en los que se van a determinar la aceptación y el rechazo son: Académico, Afectivo y Social.

Se entiende por ámbito académico las preferencias y los rechazos intragrupales para trabajar en grupo, por afectivo las preferencias y rechazos intragrupales para hacer un regalo y sociales las preferencias y los rechazos intragrupales para estar en el tiempo de ocio del recreo. 
Así, para la recogida de datos se diseñó un cuestionario sociométrico de nominación directa y con elecciones limitadas entre iguales, en el que se pide a cada alumno/a que haga dos nominaciones positivas y dos negativas de sus compañeros y compañeras de aula de acuerdo con los ámbitos académico, afectivo y social, además de razonar y justificar sus respuestas para cada una de sus elecciones.

El cuestionario de nominaciones entre iguales ha probado una adecuada fiabilidad para identificar los tipos sociométricos (Rodríguez y Morera, 2001; Warden y Mackinnon, 2003). En nuestro caso, para establecer el perfil sociométrico, hemos seguido a García Bacete $(2007,2008)$ quien analiza las nominaciones positivas y negativas recibidas a través de cálculos de probabilidad binomial continua que permiten establecer los límites superiores e inferiores de nominaciones positivas y negativas para cada uno de los ámbitos académico, afectivo y social. En la Tabla 1 se puede apreciar la propuesta de ficha técnica del cuestionario sociométrico.

El objetivo del cuestionario de nominación directa es medir la interacción intragrupal, es decir, conocer cómo se estructuran las relaciones intra-grupales dentro del aula a nivel de los ámbitos académico, afectivo y social, todos ellos en positivo y en negativo, es decir, con quién les gustaría y con quién no les gustaría realizar determinados aspectos. Además, ha de permitir determinar la autopercepción grupal que tiene de forma individual el alumno en los mismos ámbitos antes descritos.

Tabla 1. Ficha técnica del cuestionario sociométrico

\begin{tabular}{|c|c|c|c|}
\hline \multirow{4}{*}{$\begin{array}{l}\text { Características del } \\
\text { grupo }\end{array}$} & \multicolumn{3}{|l|}{ Nombre del colegio } \\
\hline & \multicolumn{3}{|l|}{ Localización } \\
\hline & \multicolumn{3}{|l|}{ Curso y grupo } \\
\hline & \multicolumn{3}{|l|}{ Profesor/ tutor } \\
\hline \multirow{3}{*}{$\begin{array}{l}\text { Características } \\
\text { internas }\end{array}$} & \multicolumn{3}{|l|}{ Listado de alumnos } \\
\hline & \multicolumn{3}{|l|}{ Antigüedad del grupo } \\
\hline & \multicolumn{3}{|l|}{ Incorporaciones/bajas } \\
\hline \multirow{8}{*}{$\begin{array}{l}\text { Características del } \\
\text { cuestionario }\end{array}$} & \multirow{3}{*}{ Criterio } & Afectivo & Regalar \\
\hline & & Académico & Trabajar en grupo \\
\hline & & Social & Estar en el recreo \\
\hline & \multirow{2}{*}{ Tipo de pregunta } & Elección & Con qué compañeros te gusta \\
\hline & & Rechazo & Con qué compañeros no te gusta \\
\hline & Posibilidades de elección & Intragrupo & Grupo clase \\
\hline & Número de elecciones & Limitada (2) & $\begin{array}{l}\text { Escribir de forma ordenada de } \\
\text { más a menos }\end{array}$ \\
\hline & Justificación & \multicolumn{2}{|c|}{ Justificar y razonar las elecciones } \\
\hline
\end{tabular}

\section{Procedimiento de validación}

Tras las sesiones de elaboración del cuestionario por parte del equipo de investigación se estableció el procedimiento a través de jueces externos y la aplicación de una prueba piloto. 


\section{Validación por jueces}

Con la validez de contenido se hace referencia a la congruencia entre los resultados del estudio y las bases teóricas que guían la investigación (Ruiz, 2014). Para ello, en nuestro cuestionario, se recurrió a un análisis del contenido y estructura, consistente en la evaluación por parte de un grupo de expertos en el área a tratar. La validez de contenido es esencial a la hora de realizar inferencias o generalizaciones a partir de los resultados que se obtendrán con el cuestionario (Escofet, Folgueiras, Luna y Palou, 2016).

En el proceso participaron ocho expertos de diferentes áreas y ámbitos educativos, a los que se les remitió el cuestionario junto con el protocolo de validación. En el protocolo de validación se especificaba el objetivo del instrumento, la descripción y una escala de valoración tipo Likert con 5 opciones, siendo 1 totalmente en desacuerdo y 5 totalmente de acuerdo, para medir aspectos específicos y aspectos globales. Los criterios sobre los que se solicitaba la valoración específica de cada una de las preguntas del instrumento eran: pertinencia con el objetivo a medir; no influencia de la redacción en la respuesta; claridad en el lenguaje empleado; adecuación del lenguaje a los encuestados e inclusión de alternativas posibles. Los criterios de valoración globales del cuestionario eran: la presentación; las instrucciones para contestar; la facilidad en la respuesta; el orden establecido; extensión o amplitud. También se dio la opción de enviar comentarios sobre el instrumento que facilitasen, llegado el caso, una nueva configuración. Una vez remitidas las respuestas al equipo de investigación se procedió a la elaboración de una nueva versión del instrumento.

\section{Prueba piloto}

Con la nueva versión del instrumento, tras las aportaciones de los expertos, se procedió a la aplicación, a través de una prueba piloto, en un aula seleccionada de forma aleatoria entre los centros de educación primaria de Burgos que iban a participar en el proyecto posterior. El objetivo fue comprobar si el alumnado al que se destina el cuestionario entendía correctamente las diferentes preguntas planteadas, si se podía realizar en un tiempo razonable, si era de interés para el alumnado y si los resultados obtenidos respondían a los objetivos para los que se plantean.

Tras los primeros contactos para acordar el momento de aplicación, se procedió a explicar al tutor y al alumnado el objetivo del cuestionario, así como de la prueba piloto. El alumnado cumplió la prueba en una sesión de unos 45 minutos de duración. El profesorado recibió con posterioridad los resultados de su grupo-clase en forma de gráficos.

Como resultado de la aplicación de la prueba piloto se acordó por parte del equipo de investigación introducir cambios en aspectos formales del cuestionario tales como: los espacios para contestar a los aspectos cualitativos de las elecciones (Figura 1) y la elaboración de un protocolo de aplicación para proporcionar la misma información en todas las aplicaciones del instrumento, así como para asegurar el procedimiento de aplicación (Tabla 2).

Los resultados del análisis de la prueba piloto fueron entregados a la tutora del aula donde se aplicó el cuestionario a través de un informe pormenorizado en el que se establecía el estatus sociométrico del alumnado, así como las justificaciones aportadas por los alumnos para sus elecciones o rechazos. 
Tabla 2. Protocolo de aplicación

\begin{tabular}{|c|c|c|}
\hline \multirow{3}{*}{ Presentación } & $\begin{array}{l}\text { Presentación del } \\
\text { equipo en el centro }\end{array}$ & $\begin{array}{l}\text { Al entrar en el centro los miembros del equipo se presentarán } \\
\text { ante el director/a del centro, con el que se ha realizado el } \\
\text { contacto previo }\end{array}$ \\
\hline & $\begin{array}{l}\text { Presentación del } \\
\text { equipo en el aula }\end{array}$ & $\begin{array}{l}\text { Equipo de investigación e innovación docente de la Univer- } \\
\text { sidad de Burgos. } \\
\text { Es necesario al menos } 2 \text { miembros del equipo para la apli- } \\
\text { cación. }\end{array}$ \\
\hline & $\begin{array}{l}\text { Presentación del } \\
\text { cuestionario }\end{array}$ & $\begin{array}{l}\text { Objetivo del cuestionario. } \\
\text { Compromiso de confidencialidad en la aplicación y en la co- } \\
\text { rrección del cuestionario por parte del equipo. }\end{array}$ \\
\hline \multirow{5}{*}{$\begin{array}{l}\text { Normas para su } \\
\text { cumplimentación }\end{array}$} & $\begin{array}{l}\text { Distribución del } \\
\text { aula }\end{array}$ & $\begin{array}{l}\text { Distribuir las mesas para un trabajo individualizado. } \\
\text { Los alumnos han de contestar de forma individualizada, sin } \\
\text { observar ni ser observado durante su realización. }\end{array}$ \\
\hline & $\begin{array}{l}\text { Personas respon- } \\
\text { sables de su apli- } \\
\text { cación }\end{array}$ & $\begin{array}{l}\text { Miembros del equipo de investigación. } \\
\text { El profesor/ tutor puede permanecer en el aula. }\end{array}$ \\
\hline & $\begin{array}{l}\begin{array}{l}\text { Personas de elec- } \\
\text { ción }\end{array} \\
\end{array}$ & $\begin{array}{l}\text { Las nominaciones sólo se pueden hacer de compañeros o } \\
\text { compañeras del aula }\end{array}$ \\
\hline & $\begin{array}{l}\text { Lectura de las pre- } \\
\text { guntas y ejemplos } \\
\text { utilizados }\end{array}$ & $\begin{array}{l}\text { Explicación por parte de los miembros del equipo de la es- } \\
\text { tructura del cuestionario. } \\
\text { En la lectura de las preguntas dar ejemplos: } \\
\text { Académico: En los trabajos en grupo del aula con qué com- } \\
\text { pañero o compañera me gusta trabajar. } \\
\text { Afectivo: de mis compañeros o compañeras a quién le haría } \\
\text { un regalo, es decir, si yo en clase hago una postal a quién se la } \\
\text { daría, no es necesario que sea un objeto comprado. } \\
\text { Social: Con quién me gusta estar en el recreo pero sólo puedo } \\
\text { elegir de mis compañeros de clase, ya que en el recreo puedo } \\
\text { jugar con compañeros y compañeras de otras clases. }\end{array}$ \\
\hline & $\begin{array}{l}\text { Obligatoriedad } \\
\text { de razonar las } \\
\text { repuestas }\end{array}$ & $\begin{array}{l}\text { Insistir en la necesidad de justificar la elección realizada en } \\
\text { cada ámbito }\end{array}$ \\
\hline \multirow{4}{*}{ Desarrollo } & $\begin{array}{l}\text { Atención a alum- } \\
\text { nado con dificul- } \\
\text { tades }\end{array}$ & $\begin{array}{l}\text { Si algún alumno o alumna presenta dificultades para su cum- } \\
\text { plimentación, uno de los miembros del equipo le ayudará }\end{array}$ \\
\hline & $\begin{array}{l}\text { Comprobación del } \\
\text { cuestionario }\end{array}$ & $\begin{array}{l}\text { Una vez finalizado el cuestionario por parte del alumnado } \\
\text { se comprobará uno por uno su correcta cumplimentación, } \\
\text { animando al alumno a subsanar los aspectos que falten }\end{array}$ \\
\hline & $\begin{array}{l}\text { Actividad tras su } \\
\text { cumplimentación }\end{array}$ & $\begin{array}{l}\text { Debido al diferente ritmo de cumplimentación. El alumnado } \\
\text { que vaya finalizando se le animará a realizar un dibujo libre } \\
\text { en la parte final del cuestionario, o bien se pactará con el tutor } \\
\text { la continuidad de la actividad académica }\end{array}$ \\
\hline & Agradecimiento & $\begin{array}{l}\text { Agradecer el tiempo invertido al alumnado y al tutor del } \\
\text { centro }\end{array}$ \\
\hline
\end{tabular}


Nombre y apellidos:

1). ¿Con qué compañeros te gusta hacer los trabajos en grupo? Escribelo por orden de más a menos.

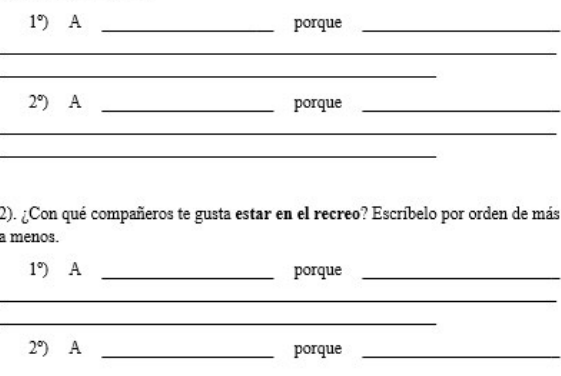

3). ¿A qué compañeros te gusta regalarle cosas? Escribelo por orden de más a menos.

$1^{\circ} \mathrm{A}$ porque

$\left.2^{\circ}\right) \mathrm{A}$ porque
4). ¿Con qué compañeros NO te gusta estar en el recreo? Escribelo por orden de más a menos.

$\left.1^{\circ}\right) \mathrm{A}$

porque

\begin{tabular}{lll}
\hline & & \\
\hline $\left.2^{\circ}\right)$ A & porque \\
\hline & \\
\hline
\end{tabular}

5). ¿Con qué compañeros NO te gusta hacer los trabajos en grupo? Escribelo por orden de más a menos.

$\left.1^{\circ}\right) \mathrm{A}$ porque

$\left.2^{\circ}\right) \mathrm{A} \longrightarrow$ porque

6). ¿A qué compañeros NO te gusta regalarle cosas? Escribelo por orden de más a menos.

$1^{\circ} \mathrm{A}$ porque

$\left.2^{\circ}\right) \mathrm{A}$ porque

GRACIAS POR TU COLABORACION

Figura 1. Cuestionario sociométrico tras la validación

En la Figura 2, se muestran los gráficos que se adjuntaron al informe en el que, por razones de confidencialidad, se ha eliminado parte de la información resultante. La valoración del informe, efectuada por la tutora del aula, fue altamente positiva y necesaria en la toma de decisiones dentro del aula.
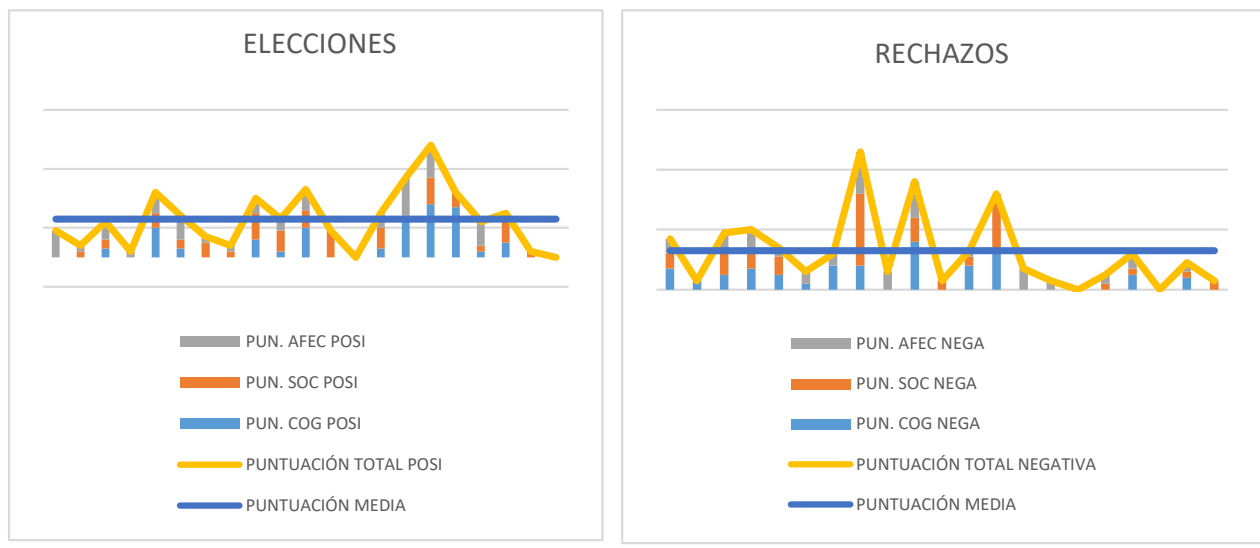

Figura 2. Resultados del análisis de los cuestionarios de la prueba piloto 


\section{Aplicación del sociograma}

Una vez efectuada la validación del cuestionario se procedió, a su aplicación en las aulas cuyos profesores, participaban en acciones de formación continua dirigida a la implantación de esta metodología. De los 35 centros inscritos en las acciones formativas del CFIE de Burgos, 11 aceptaron participar en el proyecto. En la Tabla 3, se detallan los centros participantes, la acción formativa que desarrollan, las aulas en las que se aplicó el cuestionario, así como el número total de cuestionarios aplicados.

Tabla 3. Muestra de centros de aplicación del cuestionario

\begin{tabular}{|c|c|c|c|c|c|}
\hline Centro & Acción formativa & Población & Aulas cuestionario & $\begin{array}{c}\text { Número } \\
\text { cuestionarios }\end{array}$ & Total \\
\hline $\begin{array}{c}\text { CEIP } \\
\text { Santa María }\end{array}$ & \begin{tabular}{|c|} 
Diseñar una red de \\
aprendizaje cooperativo \\
en un colegio
\end{tabular} & Aranda de Duero & $5^{\circ} \mathrm{B} / 4^{\circ} \mathrm{A} / 6^{\circ} \mathrm{A} / 6^{\circ} \mathrm{B}$ & $22+24+22+23$ & 91 \\
\hline $\begin{array}{l}\text { CEIP Alejandro Rodrí- } \\
\text { guez de Valcárcel }\end{array}$ & $\begin{array}{c}\text { Puesta en práctica del } \\
\text { aprendizaje cooperativo }\end{array}$ & Burgos & $3^{\circ} \mathrm{A} / 4^{\circ} \mathrm{A}$ & $18+21$ & 39 \\
\hline $\begin{array}{l}\text { CEIP Fernando de } \\
\text { Rojas }\end{array}$ & \begin{tabular}{|c|} 
Diseñar una red de \\
aprendizaje cooperativo \\
en un colegio
\end{tabular} & Burgos & $\begin{array}{l}\text { Los profesores } \\
\text { participantes no } \\
\text { tutorizan aulas }\end{array}$ & & \\
\hline $\begin{array}{c}\text { CEIP } \\
\text { Juan Abascal }\end{array}$ & $\begin{array}{c}\text { Puesta en práctica del } \\
\text { aprendizaje cooperativo }\end{array}$ & Briviesca & $\begin{array}{c}2^{\circ} \mathrm{A} / 2^{\circ} \mathrm{B} / 3^{\circ} \mathrm{A} / 3^{\circ} \mathrm{B} / \\
4^{\circ} \mathrm{A} / 4^{\circ} \mathrm{B} / 5^{\circ} \mathrm{A} / 5^{\circ} \mathrm{B} / \\
6^{\circ} \mathrm{A} / 6^{\circ} \mathrm{B}\end{array}$ & $\begin{array}{c}16+17+18+19+ \\
20+21+19+18+ \\
24+23\end{array}$ & 195 \\
\hline $\begin{array}{c}\text { CEIP } \\
\text { Juan de Vallejo } \\
\text { (Burgos) }\end{array}$ & $\begin{array}{c}\text { Puesta en práctica del } \\
\text { aprendizaje cooperativo }\end{array}$ & Burgos & $\begin{array}{l}3^{\circ} \mathrm{A} / 3^{\circ} \mathrm{B} / 3^{\circ} \mathrm{C} / 4^{\circ} \mathrm{A} / \\
4^{\circ} \mathrm{B} / 4^{\circ} \mathrm{C} / 5^{\circ} \mathrm{A} / 5^{\circ} \mathrm{B} / \\
5^{\circ} \mathrm{C} / 6^{\circ} \mathrm{A} / 6^{\circ} \mathrm{B} / 6^{\circ} \mathrm{C}\end{array}$ & $\begin{array}{c}25+25+25+24 \\
+24+ \\
24+27+27+26 \\
+26+23+25\end{array}$ & 301 \\
\hline $\begin{array}{c}\text { CEIP } \\
\text { Los Vadillos }\end{array}$ & $\begin{array}{c}\text { Diseñar una red de } \\
\text { aprendizaje cooperativo } \\
\text { en un colegio }\end{array}$ & Burgos & $4^{\circ} \mathrm{A}$ & 27 & 27 \\
\hline $\begin{array}{l}\text { CEIP Maximino San } \\
\text { Miguel de la Cámara }\end{array}$ & \begin{tabular}{|c|} 
Diseñar una red de \\
aprendizaje cooperativo \\
en un colegio
\end{tabular} & Gumiel de Izán & $3^{\circ}-4^{\circ} / 5^{\circ}-6^{0}$ & $11+15$ & 26 \\
\hline $\begin{array}{c}\text { CEIP Villagonzalo } \\
\text { Pedernales } \\
\end{array}$ & $\begin{array}{c}\text { Puesta en práctica del } \\
\text { aprendizaje cooperativo }\end{array}$ & $\begin{array}{l}\text { Villagonzalo } \\
\text { Pedernales } \\
\end{array}$ & $\begin{array}{l}3^{\circ} \mathrm{A} / 3^{\circ} \mathrm{B} / 4^{\circ} \mathrm{A} / 4^{\circ} \mathrm{B} / \\
5^{\circ} \mathrm{A} / 5^{\circ} \mathrm{B} / 6^{\circ} \mathrm{A} / 6^{\circ} \mathrm{B}\end{array}$ & $\begin{array}{c}18+21+22+22 \\
+19+21+17+16 \\
\end{array}$ & 156 \\
\hline $\begin{array}{l}\text { Centro Apóstol San } \\
\text { Pablo } \\
\end{array}$ & $\begin{array}{c}\text { Puesta en práctica del } \\
\text { aprendizaje cooperativo }\end{array}$ & Burgos & $3 \% 4^{\circ} / 5^{\circ} / 6^{\circ}$ & $21+24+22+20$ & 87 \\
\hline $\begin{array}{c}\text { Centro Internacional } \\
\text { Campolara }\end{array}$ & \begin{tabular}{|c|} 
Diseñar una red de \\
aprendizaje cooperativo \\
en un colegio
\end{tabular} & Burgos & $1 \% / 2^{\circ} / 3^{\circ} / 4^{\circ} / 5^{\circ} / 6^{\circ}$ & $\begin{array}{c}20+24+27+ \\
27+24+23\end{array}$ & 145 \\
\hline \multirow[t]{2}{*}{$\begin{array}{c}\text { IES } \\
\text { Camino de Santiago } \\
\end{array}$} & \begin{tabular}{|c|} 
Aprendizaje cooperati- \\
vo en secundaria
\end{tabular} & Burgos & $\begin{array}{c}1^{\circ} \mathrm{B} / 1^{\circ} \mathrm{C} / 2^{\circ} \mathrm{H} / 3^{\circ} \mathrm{A} / \\
3^{\circ} \mathrm{H} / 3^{\circ} \mathrm{B} / 4^{\circ} \mathrm{A} \\
\end{array}$ & $\begin{array}{c}25+27+10+10 \\
+14+24+26 \\
\end{array}$ & 136 \\
\hline & & & & & 1.203 \\
\hline
\end{tabular}




\section{Discusión y Conclusiones}

Para poder llevar a cabo el proyecto de investigación "Metodologías Activas y Desempeño Docente: un camino para la inclusión en las aulas" se hacía necesario diseñar un cuestionario que contemplara las tres vertientes que interesaban para la investigación. En definitiva, un instrumento que permitiera medir la interacción intragrupal del aula antes y después de la implantación del AC.

En nuestra investigación, al igual que en las realizadas por Mérida, Serrano y Tabernero (2015), Escofet, Folgueiras, Luna y Palou (2016) o Del Rey, Casa y Ortega (2017), el proceso de validación del instrumento resulta fundamental para determinar la validez de contenido, esto permitirá inferir y generalizar los resultados que se obtengan a partir del cuestionario. En nuestro caso, se procedió a su validación por ocho expertos en diferentes áreas.

Con las recomendaciones de dichos expertos se elaboró una nueva versión que pasó a aplicarse como prueba piloto y, tras comprobar su viabilidad, a los centros y aulas participantes en el proyecto (Ver Tabla 3).

Puesto que la investigación sobre la incidencia del AC en el estatus sociométrico de los alumnos se está desarrollando de forma paulatina, los resultados obtenidos, así como las conclusiones que se deriven del análisis, serán objeto de estudios posteriores.

La educación se encuentra inmersa en procesos de cambio debido a las características propias de la sociedad actual, lo que demanda la incorporación de procesos que permitan el desarrollo de nuevas competencias que exceden la mera adquisición de conocimientos. En este sentido el AC es una herramienta que incide en uno de los ámbitos que le es propio a la educación, como es la adquisición de competencias sociales, ya que a través de su estructuración de trabajo en grupo y de su objetivo centrado en desarrollar actitudes relacionales intragrupo, redunda en la mejora de la cohesión social, en este caso de aula.

Nuestra investigación aporta un instrumento que, tras el proceso de validación, pretende establecer los estatus sociométricos que se dan dentro de las aulas como punto de partida para continuar en el desarrollo del proyecto que determinará si el $\mathrm{AC}$ es una herramienta moduladora de los aspectos relacionales dentro del aula, considerando éste como un contexto dinámico, en el que los constructos sociométricos pueden llegar a aportar datos predictivos (García Bacete, 2007, 2008).

\section{Referencias Bibliográficas}

Antolín, A., Martín-Pérez, G. y Barbá, J. (2012). El aprendizaje cooperativo para la mejora de la socialización y la educación a través del conflicto. La Peonza, Revista de Educación Física para la Paz, 7, 3-11.

Antonis, M. (2017). Rendir cuentas en el ámbito de la educación. París: UNESCO.

Barber, M. y Mourshed, M. (2007). Cómo hicieron los sistemas educativos con mejor desempeño del mundo para alcanzar sus objetivos. Londres: McKinsey \& Company. 
VALIDACIÓN DE UN INSTRUMENTO QUE ESTIMA LA INCIDENCIA DEL APRENDIZAJE COOPERATIVO...

Benavot, A. (2016). La educación al servicio de los pueblos y el planeta. París: UNESCO.

Coie, J., Dodge, K. y Coppotelli, H. (1982). Dimensions and types of social status: A cross-age perspective. Developmental Psychology, 18, 557-570.

Del Rey, R., Casa, J. A. y Ortega Ruiz, R. (2017). Desarrollo y validación de la Escala de Convivencia Escolar (ECE). Universitas Psychologica, 16(1), 1-11.

Escofet, A., Folgueiras, P., Luna, E. y Palou, B. (2016). Elaboración y validación de un cuestionario para la valoración de proyectos de aprendizaje-servicio. RMEl, $21(70), 929-949$.

García Bacete, F., Sureda García, I. y Monjas Casares, M. (2010). El rechazo entre iguales en la educación primaria: Una panorámica general. Anales de Psicología, 26(1), 123-136.

García-Bacete, F. J. (2007). La identificación de alumnos rechazados, preferidos, ignorados y controvertidos en el aula. Revista de Psicología General y Aplicada, 60 (1-2), 25-46.

García-Bacete, F. J. (2008). Identificación de subtipos sociométricos en niños y niñas de 6 a 11 años. Revista Mexicana de Psicología, 25(2), 209- 222.

Gillies, R. (2014). Developments in Cooperative Learning: Review of Research. Anales de Psicología,30(3), 792-801.

Goikoetxea, E. y Pascual, G. (2002). Aprendizaje cooperativo: Bases teóricas y hallazgos empíricos que explican su eficacia. Educación XXI, 5, 227-247.

Johnson, D. y Johnson, R. (2014). Cooperative Learning in 21st Century. Anales de Psicología, 30(3), 841-851.

Kagan, S. (1995). We Can Talk: Cooperative Learning in the Elementary ESL Classroom. ERIC Digest. ERIC Custom Transformations Team, 9-14. Recuperado de https:// www.kaganonline.com/free_articles/dr_spencer_kagan/ASK40.pdf

León del Barco, B., Polo del Río, M. I. y Gozalo Delgado, M. (2016). Relevancia del aprendizaje cooperativo sobre los diferentes perfiles de la dinámica bullying: un análisis mediante pruebas de tamaño del efecto. Anales de Psicología, 32(1), 8088.

Martínez Lírola, M. (2016). How to Use Cooperative Learning for Assessing Students' Emotional Competences: A Practical Example at the Tertiary Level. Profile-Issues in Teachers Professional Development, 18(2), 153-165.

Martínez Lírola, M. (2016). Hacia una resolución efectiva de conflictos en las aulas universitarias: Ejemplos a través del debate cooperativo. Zona Próxima, 0(24), 103-114.

Mérida, R., Serrano, A. y Tabernero, C. (2015). Diseño y validación de un cuestionario para la evaluación de la autoestima en la infancia. Revista de Investigación Educativa, 33(1), 149-162. 
Muñoz, V., Moreno, M. C. y Jiménez, I. (2008). Las tipologías de estatus sociométrico durante la adolescencia: contraste de distintas técnicas y fórmulas para su cálculo. Psicothema, 20(4), 665-671.

Pliego Prenda, N. (2011). El aprendizaje cooperativo y sus ventajas en la educación intercultural. Revista Educativa Digital. Hekademos, 8, 63-76

Polo del Río, M.I., Mendo Lázaro, S., Fajardo Bullón, F. y León del Barco, B. (2017). Una intervención en aprendizaje cooperativo sobre el perfil del observador en la dinámica bullying. Universitas Psychologica, 16(1), 1-13

Reimers, F. M. y Chung, C. K. (2017). Enseñanza y aprendizaje en el S. XXI. México D.F: Fondo de Cultura Económica.

Rodríguez, A. y Morera, D. (2001). La representación sociométrica individual. En A. Rodriguez y D. Morera (Eds.). El sociograma. Estudio de las relaciones informales en las organizaciones. Madrid. Pirámide

Rohner, R. P. y Carrasco, M. A. (2014). Teoría de la Aceptación-Rechazo Interpersonal (IPARTheory): Bases conceptuales, Método y Evidencia Empírica. Acción Psicológica, 11(2), 9-26.

Ruiz, A. (2014). La operacionalización de elementos teóricos al proceso de medida. Barcelona: Universitat de Barcelona.

Slavin, R. E. (2010). Cooperative learning. En E. Baker, P. Peterson y B. McGaw (Eds.), International encyclopedia of education (3rd ed.). Oxford, England: Elsevier.

Slavin, R. (2013). Cooperative learning and achievement: Theory and research. In W. Reynolds, G. Miller e I. Weiner (Eds.) Handbook of psychology (2nd ed., Vol. 7, pp. 199-212.). Hoboken, NJ: Wiley.

Slavin, R. (2014). Cooperative Learning and Academic Achievement: Why Does Groupwork Work? Anales de Psicología, 30(3), 785-791.

Sureda García, I., García-Bacete, F. J. y Monjas Casares, M. I. (2009). Razones de niños y niñas de diez y once años para preferir o rechazar a sus iguales. Revista Latinoamericana de Psicología, 41(2), 323-334.

UNESCO (2015). Replantear la educación ¿Hacia un bien común mundial? París: UNESCO.

UNESCO (2017). La educación transforma vidas. París: UNESCO.

UNESCO (2018). Cumplir nuestros compromisos de igualdad de género en la educación. París: UNESCO

Vega Vaca, M. L., Vidal Rodríguez, D. y del Pilar García, M. (2013). Avances acerca de los efectos del aprendizaje cooperativo sobre el logro académico y las habilidades sociales en relación con el estilo cognitivo. Revista Colombiana de Educación, 64, 155-174. 
VALIDACIÓN DE UN INSTRUMENTO QUE ESTIMA LA INCIDENCIA DEL APRENDIZAJE COOPERATIVO...

Warden, D. y Mackinnon, S. (2003). Prosocial children, bullies and victims: An investigation of their sociometric status, empathy and social-problem-solving strategies. British Journal of Developmental Psychology, 21, 367-385. 